\title{
1-Benzyl-1,2,3,4-tetrahydroisoquinoline, an Endogenous Neurotoxic Compound, Disturbs the Behavioral and Biochemical Effects of L-DOPA: In Vivo and Ex Vivo Studies in the Rat
}

\author{
Agnieszka Wąsik · Irena Romańska • \\ Jerzy Michaluk • Małgorzata Kajta • \\ Lucyna Antkiewicz-Michaluk
}

Received: 17 February 2014/Revised: 23 April 2014/Accepted: 23 April 2014/Published online: 20 May 2014

(C) The Author(s) 2014. This article is published with open access at Springerlink.com

\begin{abstract}
Environmental factors and endogenously produced toxins, such as 1-benzyl-1,2,3,4-tetrahydroisoquinoline (1BnTIQ), are considered to be involved in the pathogenesis of Parkinson's disease (PD). In this study, we investigated the impact of single and multiple 1BnTIQ (25 and $50 \mathrm{mg} / \mathrm{kg}$ i.p.) administration on L-DOPA-induced changes in the rate of dopamine and serotonin metabolism in the rat brain. Additionally, using in vivo microdialysis, we measured the impact of acute and multiple 1BnTIQ administrations on L-DOPA-induced dopamine release in the striatum. These data were compared with results from behavioral tests in which we measured the effect of 1BnTIQ and L-DOPA on locomotor activity. Finally, we determined the effect of the repeated administration of 1BnTIQ on the L-DOPA-induced elevation of caspase-3 activity in the hippocampus. An ex vivo neurochemical study indicated that both acute and chronic 1BnTIQ injections strongly inhibited L-DOPA-induced increases in the concentration of dopamine and all of its metabolites in dopaminergic structures. In contrast, in vivo microdialysis studies suggested that the differences in 1BnTIQ's effects are dependent on the type of treatment. A single dose of 1BnTIQ intensified the elevation of dopamine release induced by L-DOPA administration $(\sim 1,300 \%$; $P<0.01$ ), while multiple administrations of 1BnTIQ significantly enhanced the basal dopamine levels while
\end{abstract}

A. Wąsik $(\bowtie) \cdot$ I. Romańska · J. Michaluk ·

L. Antkiewicz-Michaluk

Department of Neurochemistry, Institute of Pharmacology Polish Academy of Sciences, 12 Smetna Street, 31-343 Kraków, Poland e-mail: wasik@if-pan.krakow.pl

M. Kajta

Department of Neuroendocrinology, Institute of Pharmacology

Polish Academy of Sciences, 31-343 Kraków, Poland partially diminishing the effects of L-DOPA injection ( $\sim 200 \% ; P<0.01)$. Additionally, we found that chronic administration of 1 BnTIQ completely blocked the L-DOPA-induced increase in caspase- 3 activity in the hippocampus. These findings indicate that both acute and chronic administrations of 1BnTIQ disturbs the behavioral and biochemical effects of L-DOPA in the rat. The data presented from ex vivo and in vivo studies clearly suggest that 1BnTIQ's effects may be connected with the inhibition of DAT and/or COMT activity in the brain. Furthermore, elevated endogenous levels of 1 BnTIQ may pose a serious risk in PD patients undergoing L-DOPA therapy.

Keywords 1-Benzyl-1,2,3,4-tetrahydroisoquinoline · L-DOPA · Parkinson's disease - Microdialysis study · Caspase-3 activity $\cdot$ Dopamine

\section{Introduction}

Parkinson's disease (PD) is an age-related movement disorder characterized by a progressive loss of dopaminergic neurons of the substantia nigra and is associated with postural and behavioral abnormalities, such as bradykinesia, tremors, and rigidity (Klockgether 2004). In spite of intensive research efforts, the cause of dopaminergic cell neurodegeneration in PD remains unknown. Some factors that may be responsible for the degeneration of dopaminergic neurons are oxidative stress, mitochondrial dysfunction, protein misfolding, apoptosis, and inflammation (Gandhi and Wood 2005; Moore et al. 2005; Sas et al. 2007). Dopaminergic brain structures are particularly sensitive to oxidative stress because the metabolism of dopamine itself leads to the generation of reactive oxygen species (ROS). ROS initiate the mitochondrial-caspase 
cascade, which leads to the activation of the main effector, caspase-3 (Hanrott et al. 2006; Bayir et al. 2009). Currently, L-DOPA is the best treatment available for improving the symptoms of PD. L-DOPA therapy is required by all $\mathrm{PD}$ patients at some stage in their illness.

The pathogenesis of PD may involve both environmental factors and endogenous toxins. 1-Benzyl-1,2,3, 4-tetrahydroisoquinoline (1BnTIQ), an endogenous neurotoxin, has been proposed as a possible etiological factor of idiopathic PD (Kotake et al. 1995). The concentration of 1BnTIQ in the CSF of parkinsonian patients is three times higher than that in the CSF of neurological control subjects (Kotake et al. 1995). Chronic treatment with 1BnTIQ produces parkinsonianlike symptoms in rodents and primates (Kotake et al. 1995, 2003; Kohta et al. 2010). Evidence demonstrates that 1 BnTIQ induces cell death via apoptosis and dosedependently elevates the level of the pro-apoptotic protein Bax, while decreasing the concentration of the antiapoptotic protein Bcl-xl. Additionally, 1BnTIQ produces an increase in the formation of the active caspase-3 protein fragments (Shavali and Ebadi 2003). 1BnTIQ, which is synthesized endogenously in the brain and/or is obtained exogenously in the diet, can be taken up by neurons via dopamine transporter (DAT) (Okada et al. 1998). As such, 1 BnTIQ accumulates in dopaminergic neurons, where it is thought to exert some pathological effects leading to Parkinsonism. In previous studies, we showed that 1BnTIQ significantly affects dopamine (DA) structures, producing an increase in the rate of DA metabolism together with pronounced activation of the oxidative MAO-dependent catabolic pathway (Antkiewicz-Michaluk et al. 2001; Wasik et al. 2009). 1BnTIQ also significantly inhibits the COMT-dependent O-methylation pathway. The depression of DA levels produced by $1 \mathrm{BnTIQ}$ is most pronounced in the striatum and nucleus accumbens because this effect is specific to dopaminergic neurons.

The aim of this study was to determine the consequences of acute and chronic administrations of 1BnTIQ on the effects of L-DOPA in the rat brain using behavioral and biochemical assays. We used the dose of L-DOPA which produces light stimulation with simultaneously significant biochemical changes. In neurochemical experiments, we investigated L-DOPA-induced changes in the rate of DA metabolism, as well as in vivo DA release in different brain structures after treatment with 1BnTIQ. The effects of 1BnTIQ on L-DOPA metabolism in the striatum and L-DOPA-induced elevation of caspase-3 activity in the hippocampus were also examined. In behavioral studies, we measured the effects of acute and chronic administrations of 1BnTIQ on L-DOPA-induced locomotor hyperactivity.

\section{Materials and Methods}

Animals and Treatments

All experiments were carried out in male Wistar rats with an initial body weight of 220-240 g. All animals had free access to standard laboratory food and tap water and were kept at room temperature $\left(22{ }^{\circ} \mathrm{C}\right)$ under an artificial light/ dark cycle $(12 / 12 \mathrm{~h}$, light on at 7:00).

1-Benzyl-1,2,3,4-tetrahydroisoquinoline (1BnTIQ) was administered at a dose of 25 or $50 \mathrm{mg} / \mathrm{kg}$ intraperitoneally (i.p.) once or chronically for 14 consecutive days. In the mixed group, L-DOPA (100 mg/kg i.p.) was administered once, 15 min after the last 1BnTIQ administration. Control rats were treated with the appropriate vehicle. Rats were killed by decapitation $2 \mathrm{~h}$ after last drug injections and different brain structures were dissected for analysis. The experiments were carried out between 9:00 and 16:00.

All experimental procedures were carried out in accordance with the National Institutes of Health Guide for the Care and Use of Laboratory Animals and were granted an approval from the Bioethics Commission as compliant with Polish Law. All experimental procedures were approved by the Local Bioethics Commission of the Institute of Pharmacology, Polish Academy of Sciences in Kraków.

\section{Drugs}

1-Benzyl-1,2,3,4-tetrahydroisoquinoline (1BnTIQ hydrochloride) was synthesized (according to Cannon and Webster 1957) at the Department of Drug Chemistry of the Institute of Pharmacology, the Polish Academy of Sciences in Krakow. Purity of the compound was verified by measurement of the melting point and homogeneity was assessed on a chromatographic column. L-DOPA (SigmaAldrich, USA) was obtained commercially. The compounds were dissolved in a $0.9 \% \mathrm{NaCl}$ solution.

Behavioral Study

\section{Locomotor Activity}

Locomotor activity was assessed in actometers (OptoVarimex activity monitors; Columbus Inst., USA) linked on-line to a compatible IBM PC. Each cage $(43 \times 44 \times 25 \mathrm{~cm})$ perimeter was lined with an array of $15 \times 15$ photocell beams located $3 \mathrm{~cm}$ from the floor surface as reported previously (Filip et al. 2007). Interruptions of the photocell beams were counted as a measure of horizontal locomotor activity and were defined as the distance traveled (in $\mathrm{cm}$ ). Horizontal locomotor activity was recorded for 60 (acute treatment group) or $90 \mathrm{~min}$ (14-day chronic treatment group) and analyzed using the 
Auto-Track Software Program (Columbus Instruments, USA). Animals were placed into the actometers for a 15-min adaptation period before drug administration and behavioral testing. Rats were given 25 or $50 \mathrm{mg} / \mathrm{kg} 1 \mathrm{BnTIQ}$ i.p. either as their only acute dose (acute treatment group) or as their last dose (14-day chronic treatment group). L-DOPA $(100 \mathrm{mg} / \mathrm{kg}$ i.p.) was given acutely $15 \mathrm{~min}$ after $1 \mathrm{BnTIQ}$ administration. Each group consisted of five to six animals.

\section{Biochemical Studies}

\section{Ex Vivo Experiments}

DA Metabolism and L-DOPA Metabolism Two hours after the last $1 \mathrm{BnTIQ}$ injection, rats were killed by decapitation, and the substantia nigra and striatum were immediately dissected. The tissue was frozen on dry ice $\left(-70{ }^{\circ} \mathrm{C}\right)$ until use in a biochemical assay. The levels of DA and its metabolites, 3,4-dihydroxyphenylacetic acid (DOPAC), 3-methoxytyramine (3-MT), and homovanillic acid (HVA), as well as the L-DOPA metabolite, 3-methoxy-DOPA (3-MDOPA) were assayed by highperformance liquid chromatography (HPLC) with electrochemical detection (Hewlett Packard 1049A). The tissue samples were weighed and homogenized in ice-cold $0.1 \mathrm{M}$ perchloroacetic acid containing $0.05 \mathrm{mM}$ ascorbic acid. After centrifugation $(10,000 \times g$ for $5 \mathrm{~min})$, the supernatants were filtered through RC 58 0.2-im cellulose membranes (Bioanalytical Systems, West Lafayette, IN, USA). The HP 1050 chromatograph (Hewlett-Packard, Golden, CO, USA) was equipped with $\mathrm{C} 18$ columns. The electrochemical cell potential was $800 \mathrm{mV}$. The mobile phase consisted of $0.05 \mathrm{M}$ citrate-phosphate buffer ( $\mathrm{pH} 3.5$ ), $0.1 \mathrm{mM}$ EDTA, $1 \mathrm{mM}$ sodium octyl sulfonate, and $3.5 \%$ methanol. The flow rate was maintained at $1 \mathrm{ml} / \mathrm{min}$. DA and its metabolites were quantified by chromatograph peak height in comparison with standards run on the day of analysis. Each group consisted of five to six animals.

Assessment of Caspase-3 Activity Caspase-3 activity was determined according to Nicholson et al. (1995). 1BnTIQ was administered chronically at a concentration of 25 or $50 \mathrm{mg} / \mathrm{kg}$ i.p. for 14 consecutive days. In the mixed group, L-DOPA (100 mg/kg i.p.) was given once, $15 \mathrm{~min}$ after last 1BnTIQ administration. Rats were decapitated $3 \mathrm{~h}$ after the last injection. The assessment of caspase-3 activity was performed as previously described (Kajta et al. 2009). Cell lysates were incubated at $36{ }^{\circ} \mathrm{C}$ with a colorimetric substrate, Ac-DEVD- $p$ NA ( $N$-acetyl-asp-glu-val-asp- $p$-nitroanilide), which is preferentially cleaved by caspase- 3 . The absorbance of Ac-DEVD- $p$ NA was monitored continuously over 60 min with a Multiskan Spectrum Microplate Spectrophotometer (ThermoLabsystems, Vantaa, Finland).
Data were analyzed with Ascent software, normalized to the absorbance in vehicle-treated cells, and expressed as the percent of control \pm SEM of three to four independent experiments. The absorbance of blanks, acting as noenzyme controls, was subtracted from each value.

\section{In Vivo Microdialysis}

Rats were anesthetized with ketamine $(75 \mathrm{mg} / \mathrm{kg})$ and xylazine $(10 \mathrm{mg} / \mathrm{kg})$ and secured in a stereotaxic frame (Stoelting, USA). Vertical microdialysis guide cannulas (Intracerebral Guide Cannula with stylet; BAS Bioanalytical, USA) were implanted into the striatum (STR) according to the following stereotaxic coordinates: $\mathrm{A} / \mathrm{P}$ $+1.0, \mathrm{~L} / \mathrm{M}+2.5$, and V/D $-3.5 \mathrm{~mm}$ from bregma and dura (G. Paxinos and C. H. Watson). 7 days after surgery, microdialysis probes were inserted into the cannulas, and the striatum was perfused with an artificial cerebrospinal fluid (aCSF) consisting of $140 \mathrm{mM} \mathrm{NaCl}, 2.7 \mathrm{mM} \mathrm{KCl}$, $1.2 \mathrm{mM} \mathrm{CaCl}_{2}, 1 \mathrm{mM} \mathrm{MgCl}_{2}, 0.3 \mathrm{mM} \mathrm{NaH} \mathrm{PO}_{4}$, and $1.7 \mathrm{mM} \mathrm{Na}_{2} \mathrm{HPO}_{4}(\mathrm{pH} 7.4)$ at a flow rate of $1.5 \mu \mathrm{l} / \mathrm{min}$ with a microinfusion pump (Stoelting, IL, USA). Samples were collected from freely moving rats in 20-min intervals after a 3-h wash-out period. 1BnTIQ was injected (acute or chronic: for 14 consecutive days) in a $50 \mathrm{mg} / \mathrm{kg}$ i.p. and dialysis samples were collected for $180 \mathrm{~min}$. In the mixed groups, L-DOPA (100 mg/kg i.p.) was administered $40 \mathrm{~min}$ after the last 1BnTIQ injection. All dialysates were immediately frozen on dry ice $\left(-70{ }^{\circ} \mathrm{C}\right)$ until use in a biochemical assay.

Levels of DA and its extraneuronal metabolite, 3-MT, were assayed in dialysates $(20 \mu \mathrm{l})$ using HPLC with electrochemical detection as described above.

Chromatographic data were processed using the ChemStation computer program (Hewlett Packard, USA). DA and its metabolites were quantified by chromatograph peak height in comparison with standards run on the day of analysis. At the end of the experiment, frozen brains were examined histologically for correct probe placement. Each group consisted of six animals.

\section{Calculations and Statistics}

A two-way analysis of variance (ANOVA) for repeated measures was used to analyze the results of the behavioral test (locomotor activity). Differences between control and experimental groups were assessed with Duncan's post hoc test. Data from the microdialysis study (acute 1BnTIQ treatment) were analyzed by a one-way ANOVA for repeated measures. The results from experiments evaluating chronic 1BnTIQ administration were analyzed by a two-way ANOVA for repeated measures, followed by (if significant differences arose) Duncan's post hoc test. 
Fig. 1 The effect of acute administration of 1BnTIQ on LDOPA-induced hyperactivity in rats. Rats were placed into actometers and received treatments after $15 \mathrm{~min}$ of adaptation. Rats received a single injection of saline (control), 1BnTIQ [25 mg/kg i.p. (a) or $50 \mathrm{mg} / \mathrm{kg}$ i.p. (b)] or L-DOPA (100 mg/kg i.p.). In the mixed group, L-DOPA was injected $15 \mathrm{~min}$ after 1BnTIQ administration. Movements were recorded for $60 \mathrm{~min}$. The data are expressed as the mean \pm SEM $(n=5-6$ animals). Data were analyzed with a two-way ANOVA for repeated measures, followed by Duncan's post-hoc test.

Statistical significance: ${ }^{*} P<0.05,{ }^{*} * P<0.01$ versus saline-treated group; ${ }^{+} P<0.05$ versus L-DOPA-treated group
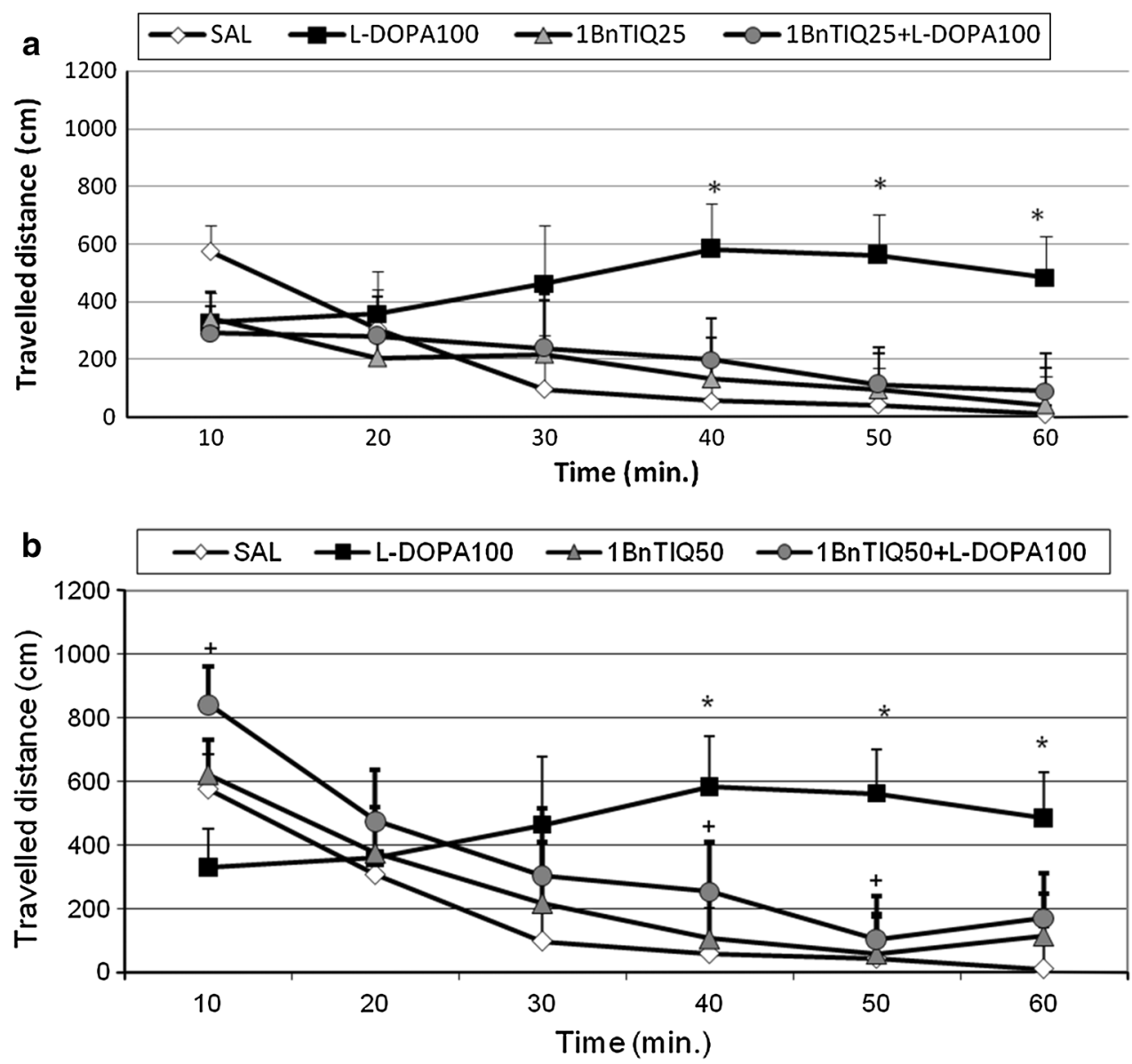

The results of the biochemical experiments were analyzed by a two-way ANOVA, followed, when appropriate, by Duncan's post hoc test. The total catabolism rate of DA was calculated from the ratio of the concentration of the final DA metabolite, HVA, to the concentration of DA and was expressed as a catabolism rate index ([HVA]/ [DA] $\times 100$ ), as previously described in detail (Antkiewicz-Michaluk et al. 2001). These indices were calculated using the concentrations of individual tissue samples $(n=6)$.

\section{Results}

Behavioral Study

The Effect of Acute 1BnTIQ Administration on L-DOPAInduced Hyperactivity in Rats

L-DOPA (100 mg/kg i.p.) produced a significant increase in the horizontal locomotor activity of rats $(P<0.05)$ from 40 to $60 \mathrm{~min}$. In the same time frame, 1BnTIQ given alone ( $25 \mathrm{mg} / \mathrm{kg}$ i.p.) did not change locomotor activity (Fig. 1a).
In the mixed group, 1BnTIQ completely antagonized L-DOPA-induced hyperactivity.

Similarly, acute administration of 1BnTIQ at a higher dose of $50 \mathrm{mg} / \mathrm{kg}$ i.p. did not change locomotor activity, but completely antagonized L-DOPA-induced hyperactivity (Fig. 1b).

The Effect of Chronic 1BnTIQ Administration on L-DOPAInduced Hyperactivity in Rats

The chronic (14-day) administration of 1BnTIQ at a low dose of $25 \mathrm{mg} / \mathrm{kg}$ i.p. reduced $(P<0.05)$ locomotor activity only during the first $10 \mathrm{~min}$ of measurement (Fig. 2a). L-DOPA (100 mg/kg i.p.) produced a significant increase in horizontal locomotor activity $(P<0.01)$ from 30 to $70 \mathrm{~min}$. In the mixed group, 1BnTIQ completely antagonized L-DOPA-induced hyperactivity.

Chronic 1BnTIQ administration at a higher dose of $50 \mathrm{mg} / \mathrm{kg}$ i.p. did not alter locomotor activity. The increase in locomotor activity observed with L-DOPA administration $(100 \mathrm{mg} / \mathrm{kg}$ i.p.) was completely antagonized by coadministration of $50 \mathrm{mg} / \mathrm{kg}$ 1BnTIQ following chronic treatment (Fig. 2b). 

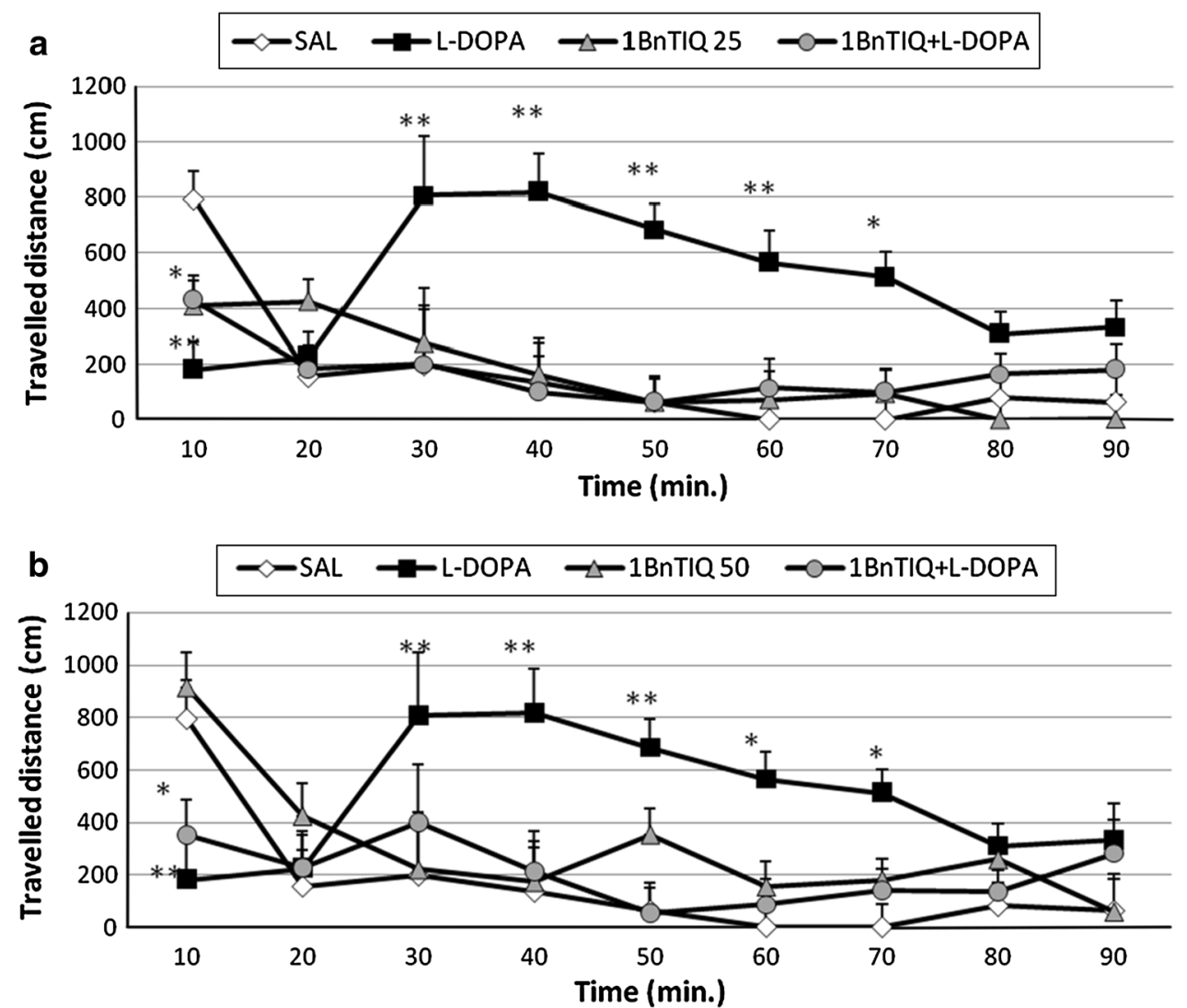

Fig. 2 The influence of chronic administration of 1BnTIQ on LDOPA-induced hyperactivity in rats. The influence of chronic administration of 1BnTIQ on L-DOPA-induced hyperactivity in rats. The rats were placed into actometers and after $15 \mathrm{~min}$. adaptation received drugs: 1 BnTIQ was administered at two doses $[25 \mathrm{mg} / \mathrm{kg}$ i.p. (a) and $50 \mathrm{mg} / \mathrm{kg}$ i.p (b) chronic during 14 consecutive days]. In the mixed group, L-DOPA (100 mg/kg i.p.) was given once, $15 \mathrm{~min}$ after last $1 \mathrm{BnTIQ}$ administration. The rats received a single injection of saline (control). Next the measurement was recorded during $90 \mathrm{~min}$. The data are mean \pm SEM, the number of animals was $n=5-6$; The data were analyzed by means of two-way ANOVA for repeated measures, followed when appropriate, by Duncan's post-hoc test. Statistical significance: ${ }^{*} P<0.05,{ }^{*} P<0.01$ versus control group; ${ }^{+} P<0.05$ versus L-DOPA-treated group
Biochemical Studies: Ex Vivo Experiments

\section{The Effect of Acute 1 BnTIQ Administration on L-DOPA- Induced Changes on DA Metabolism in Rat Brain Structures}

Substantia Nigra A two-way ANOVA indicated a significant effect of acute treatment with 1BnTIQ $(F[2,29]=10.8, P<0.01)$ and L-DOPA $(F[2,29]=10.7$, $P<0.01)$ on the DA concentration in the substantia nigra (Table 1). An interaction between 1BnTIQ and L-DOPA treatment was also significant $(F[2,29]=8.55, P<0.01)$. Duncan's post hoc analysis demonstrated that administration of L-DOPA strongly increased the level of DA (approximately $800 \% ; P<0.01$ ). In contrast, both concentrations of 1BnTIQ produced reductions in DA concentration. 1BnTIQ co-administered with L-DOPA completely antagonized L-DOPA-induced increases in DA (the DA level remained just below the control level) (Table 1).

A two-way ANOVA demonstrated a significant effect of 1BnTIQ $(F[2,29]=13.5, P<0.01)$, as well as L-DOPA $(F[2,29]=16.5, \quad P<0.01) \quad$ on the concentration of DOPAC in the substantia nigra (Table 1). An interaction between 1BnTIQ and L-DOPA was also significant $(F[2,29]=13.4, P<0.01)$. The post hoc analysis indicated that acute administration of L-DOPA produced massive elevation of DOPAC concentration (approximately $10,000 \% ; P<0.01$ ), and this effect was strongly blocked by both concentrations of 1BnTIQ (Table 1).

The statistical analysis showed a significant effect of 1BnTIQ $\quad(F[2,29]=19.3, \quad P<0.01) \quad$ and $\quad$ L-DOPA $(F[2,29]=28.9, P<0.01)$ treatment on the levels of 3-MT, as well as an interaction between 1BnTIQ and L-DOPA $(F[2,29]=10.1, P<0.01)$ (Table 1$)$. Duncan's post hoc test showed that L-DOPA induced an increase in 
Table 1 The effects of acute administration of 1BnTIQ on L-DOPA-induced changes in DA metabolism in rat brain structures

\begin{tabular}{|c|c|c|c|c|c|c|}
\hline Treatment acute & Treatment acute & DA (ng/g tissue) & DOPAC (ng/g tissue) & 3-MT (ng/g tissue) & HVA (ng/g tissue) & HVA/DA \\
\hline \multicolumn{7}{|l|}{ Substantia Nigra } \\
\hline Saline & Saline & $938 \pm 100$ & $216 \pm 17$ & $45 \pm 4$ & $111 \pm 10$ & $12 \pm 1$ \\
\hline Saline & L-DOPA 100 & $7438 \pm 2071 * *$ & $20649 \pm 5208^{* *}$ & $162 \pm 25^{* *}$ & $4701 \pm 695^{* *}$ & $93 \pm 36^{*}$ \\
\hline 1BnTIQ 25 & Saline & $534 \pm 67^{++}$ & $147 \pm 10^{++}$ & $24 \pm 4^{++}$ & $91 \pm 5^{++}$ & $18 \pm 2$ \\
\hline 1BnTIQ 50 & Saline & $566 \pm 47^{++}$ & $171 \pm 27^{++}$ & $27 \pm 4^{++}$ & $101 \pm 15^{++}$ & $18 \pm 2$ \\
\hline 1BnTIQ 25 & L-DOPA 100 & $869 \pm 108^{++}$ & $873 \pm 273^{++}$ & $54 \pm 13^{++}$ & $755 \pm 300^{++}$ & $88 \pm 33$ \\
\hline 1BnTIQ 50 & L-DOPA 100 & $774 \pm 93^{++}$ & $1013 \pm 493^{++}$ & $44 \pm 6^{++}$ & $935 \pm 466^{++}$ & $108 \pm 40^{*}$ \\
\hline \multicolumn{2}{|c|}{ Effect of 1BnTIQ } & $\begin{array}{l}F_{(2 / 29)}=10.8 \\
P<0.01\end{array}$ & $\begin{array}{l}F_{(2 / 29)}=13.5 \\
P<0.01\end{array}$ & $\begin{array}{l}F_{(2 / 29)}=19.3 \\
P<0.01\end{array}$ & $\begin{array}{l}F_{(2 / 29)}=18.7 \\
P<0.01\end{array}$ & $\begin{array}{l}F_{(2 / 29)}=0.1 \\
\text { N.S. }\end{array}$ \\
\hline \multicolumn{2}{|c|}{ Effect of L-DOPA } & $\begin{array}{l}F_{(2 / 29)}=10.7 \\
P<0.01\end{array}$ & $\begin{array}{l}F_{(2 / 29)}=16.5 \\
P<0.01\end{array}$ & $\begin{array}{l}F_{(2 / 29)}=28.9 \\
P<0.01\end{array}$ & $\begin{array}{l}F_{(2 / 29)}=45.6 \\
P<0.01\end{array}$ & $\begin{array}{l}F_{(2 / 29)}=14.8 \\
P<0.01\end{array}$ \\
\hline \multicolumn{2}{|c|}{ Interaction of 1BnTIQ + L-DOPA } & $\begin{array}{l}F_{(2 / 29)}=8.55 \\
P<0.01\end{array}$ & $\begin{array}{l}F_{(2 / 29)}=13.4 \\
P<0.01\end{array}$ & $\begin{array}{l}F_{(2 / 29)}=10.1 \\
P<0.01\end{array}$ & $\begin{array}{l}F_{(2 / 29)}=18.4 \\
P<0.01\end{array}$ & $\begin{array}{l}F_{(2 / 29)}=0.1 \\
\text { N.S. }\end{array}$ \\
\hline \multicolumn{7}{|l|}{ Striatum } \\
\hline Saline & Saline & $8691 \pm 265$ & $1098 \pm 43$ & $380 \pm 7$ & $713 \pm 26$ & $8.2 \pm 0.3$ \\
\hline Saline & L-DOPA 100 & $25191 \pm 3967 * *$ & $23040 \pm 6005^{* *}$ & $281 \pm 26^{* *}$ & $6238 \pm 944 * *$ & $28.1 \pm 7.9 *$ \\
\hline 1BnTIQ 25 & Saline & $6976 \pm 389^{++}$ & $1428 \pm 132^{++}$ & $348 \pm 25^{+}$ & $1065 \pm 176^{++}$ & $15.3 \pm 2.4$ \\
\hline 1BnTIQ 50 & Saline & $5246 \pm 411^{++}$ & $1187 \pm 86^{++}$ & $271 \pm 8 * *$ & $907 \pm 137^{++}$ & $18.2 \pm 3.7$ \\
\hline 1BnTIQ 25 & L-DOPA 100 & $6669 \pm 389^{++}$ & $2021 \pm 373^{++}$ & $284 \pm 12^{* *}$ & $1865 \pm 414^{++}$ & $28 \pm 5.6^{*}$ \\
\hline 1BnTIQ 50 & L-DOPA 100 & $5945 \pm 646^{++}$ & $2330 \pm 641^{++}$ & $252 \pm 17 * *$ & $2469 \pm 755^{*++}$ & $41.8 \pm 10.5^{* *}$ \\
\hline \multicolumn{2}{|c|}{ Effect of 1BnTIQ } & $\begin{array}{l}F_{(2 / 29)}=26.7 \\
P<0.01\end{array}$ & $\begin{array}{l}F_{(2 / 29)}=11.1 \\
P<0.01\end{array}$ & $\begin{array}{l}F_{(2 / 29)}=8.5 \\
P<0.01\end{array}$ & $\begin{array}{l}F_{(2 / 29)}=8.5 \\
P<0.01\end{array}$ & $\begin{array}{l}F_{(2 / 29)}=2.0 \\
\text { N.S. }\end{array}$ \\
\hline \multicolumn{2}{|c|}{ Effect of L-DOPA } & $\begin{array}{l}F_{(2 / 29)}=16.0 \\
P<0.01\end{array}$ & $\begin{array}{l}F_{(2 / 29)}=14.3 \\
P<0.01\end{array}$ & $\begin{array}{l}F_{(2 / 29)}=17.4 \\
P<0.01\end{array}$ & $\begin{array}{l}F_{(2 / 29)}=35.8 \\
P<0.01\end{array}$ & $\begin{array}{l}F_{(2 / 29)}=13.8 \\
P<0.01\end{array}$ \\
\hline \multicolumn{2}{|c|}{ Interaction of 1BnTIQ + L-DOPA } & $\begin{array}{l}F_{(2 / 29)}=15.2 \\
P<0.01\end{array}$ & $\begin{array}{l}F_{(2 / 29)}=11.5 \\
P<0.01\end{array}$ & $\begin{array}{l}F_{(2 / 29)}=2.5 \\
\text { N.S. }\end{array}$ & $\begin{array}{l}F_{(2 / 29)}=11.2 \\
P<0.01\end{array}$ & $\begin{array}{l}F_{(2 / 29)}=0.4 \\
\text { N.S. }\end{array}$ \\
\hline
\end{tabular}

1BnTIQ was acutely administered at two concentrations (25 and $50 \mathrm{mg} / \mathrm{kg}$ i.p.). In the mixed group, L-DOPA (100 mg/kg i.p.) was given once, $15 \mathrm{~min}$ after 1BnTIQ administration. Rats were decapitated $2 \mathrm{~h}$ after the injections. The results are expressed as the mean \pm SEM $(n=5-6$ animals per group). Data were analyzed with a two-way ANOVA followed by Duncan's post hoc test. Statistical significance: $* P<0.05, * *$ $P<0.01$ versus saline-treated group; ${ }^{+} P<0.05,{ }^{++} P<0.01$ versus L-DOPA-treated group

the concentration of 3-MT (approximately $300 \%$, $P<0.01)$; 1BnTIQ completely antagonized this effect (Table 1).

Treatment with 1BnTIQ $(F[2,29]=18.7, P<0.01)$ and L-DOPA $(\mathrm{F}[2,29]=45.6, \quad P<0.01)$ had a significant effect on the HVA concentration in the substantia nigra (Table 1). This analysis also revealed a significant interaction between 1BnTIQ and L-DOPA $(F[2,29]=18.4$, $P<0.01)$. The post hoc analysis showed that L-DOPA administration strongly increased the level of HVA (approx. 4,000\%,P<0.01), and this effect was weakened by both concentrations of 1BnTIQ (Table 1).

L-DOPA $(F[2,29]=14.8, P<0.01)$ also had a significant effect on the rate of DA metabolism, measured as [HAV]/[DA] (Table 1). The statistical analysis revealed no effect of 1BnTIQ on the rate of DA metabolism $(F[2,29]=0.1$, N.S. $)$ or interaction between 1BnTIQ and L-DOPA $(F[2,29]=0.1$, N.S. $)$. Duncan's post hoc analysis demonstrated that acute doses of L-DOPA strongly increased the rate of DA metabolism (approximately $800 \% ; P<0.01)$, and a similar effect was observed when $1 \mathrm{BnTIQ}(50 \mathrm{mg} / \mathrm{kg})$ was given in combination with L-DOPA.

\section{Striatum}

In the striatum, a two-way ANOVA indicated a significant effect of both 1BnTIQ $(F[2,29]=26.7, P<0.01)$ and L-DOPA $(F[2,29]=16.0, P<0.01)$ treatments on the DA concentration (Table 1). An interaction between 1BnTIQ and L-DOPA was also revealed by the analysis $(F[2,29]=15.2, \quad P<0.01)$. Duncan's post hoc test showed that acute dose of L-DOPA increased the DA concentration (approx. $300 \%, P<0.01$ ). Both concentrations of 1BnTIQ completely antagonized this effect (Table 1). 
The statistical analysis showed a significant effect of 1BnTIQ $\quad(F[2,29]=11.1, \quad P<0.01) \quad$ and $\quad$ L-DOPA $(F[2,29]=14.3, P<0.01)$ treatments on DOPAC levels, as well as an interaction between 1BnTIQ and L-DOPA $(F[2,29]=11.5, P<0.01)$ (Table 1$)$. The post hoc analysis demonstrated that treatment with L-DOPA induced a massive increase in the level of DOPAC (approximately $2,000 \%$ ). This L-DOPA-induced effect was completely blocked by 1 BnTIQ administration (Table 1).

A two-way ANOVA further revealed a significant effect of acute administration of 1BnTIQ $(F[2,29]=8.5$, $P<0.01)$ or L-DOPA $(F[2,29]=17.4, P<0.01)$ on 3-MT concentration in the striatum (Table 1). However, no interaction between 1BnTIQ and L-DOPA was detected in this case $(F[2,29]=2.5$, N.S.). Duncan's post hoc test showed that an acute dose of L-DOPA or 1BnTIQ (50 $\mathrm{mg} / \mathrm{kg}$ ) reduced the levels of 3-MT (approximately $30 \%$ ). A similar effect was observed in the mixed groups.

The statistical analysis indicated a significant effect of 1BnTIQ $(F[2,29]=8.5, \quad P<0.01)$ and L-DOPA $(F[2,29]=35.8, P<0.01)$ treatments on HVA levels, as well as an interaction between 1BnTIQ and L-DOPA $(F[2,29]=11.2, P<0.01)$. The post hoc analysis showed that treatment with L-DOPA induced a strong elevation in the level of HVA (approximately $900 \%$ ). This effect was partially antagonized by 1BnTIQ administration (Table 1).

L-DOPA also had a significant effect $(F[2,29]=13.8$, $P<0.01)$ on the rate of DA metabolism measured as [HAV]/[DA] (Table 1). The statistical analysis revealed no effect of 1BnTIQ on the rate of DA metabolism $(F[2,29]=2.0$, N.S. $)$ or interaction between 1BnTIQ and L-DOPA $(F[2,29]=0.4$, N.S. $)$. Duncan's post hoc analysis demonstrated that acute administration of L-DOPA increased the rate of DA metabolism (approximately $350 \% ; P<0.05)$; the strongest effect was observed when 1BnTIQ (50 mg/kg) was administered concomitantly with L-DOPA (approximately $500 \%$ ) (Table 1).

The effect of chronic administration of $1 B n T I Q$ on L-DOPA-induced changes on DA metabolism in rat brain structures.

\section{Substantia Nigra}

In the substantia nigra, a two-way ANOVA revealed no effect of chronic treatment with 1 BnTIQ $(F[2,30]=2.08$, N.S.) on the DA concentration (Table 2). The statistical analysis indicated a significant effect of treatment with L-DOPA $(F[2,30]=12.64, P<0.01)$ on the level of DA. There was no significant interaction between the chronic administration of $1 \mathrm{BnTIQ}$ and the acute dose of L-DOPA $(F[2,30]=2.00$, N.S. $)$. Duncan's post hoc test showed that the acute administration of L-DOPA produced an increase in the DA concentration (approximately 1,000\%; $P<0.01$ ). Additionally, when 1BnTIQ (50 mg/kg) given chronically was coupled with an acute dose of L-DOPA, a significant increase in the level of DA was observed (approx. $700 \% ; P<0.05$ ) (Table 2).

A two-way ANOVA demonstrated a significant effect of chronic treatment with 1BnTIQ $(F[2,30]=8.99$, $P<0.01)$, as well as acute L-DOPA administration $(F[2,30]=25.72, P<0.01)$ on the DOPAC concentration in the substantia nigra (Table 2). An interaction between 1BnTIQ and L-DOPA was also detected $(F[2,30]=8.94$, $P<0.01)$. The post hoc analysis showed that L-DOPA produced a massive increase in the DOPAC concentration (approximately 10,000 \%; $P<0.01$ ); this effect was partially blocked by chronic treatment with 1BnTIQ (Table 2).

The statistical analysis revealed no effect of chronic treatment with 1BnTIQ $(F[2,30]=2.22$, N.S. $)$ on the level of 3-MT (Table 2). Treatment with L-DOPA $(F[2,30]=34.2, P<0.01)$ significantly affected the $3-\mathrm{MT}$ concentration. There was no interaction between chronic administration of $1 \mathrm{BnTIQ}$ and acute administration of L-DOPA $(F[2,30]=2.55$, N.S. $)$. Duncan's post hoc test showed that acute doses of L-DOPA produced an increase in 3-MT concentration (approximately $500 \%$; $P<0.01$ ). When 1BnTIQ (50 mg/kg) given chronically was coupled with an acute dose of L-DOPA, a significant elevation in the levels of 3 -MT (approx. $400 \% ; P<0.01$ ) was also observed (Table 2).

A two-way ANOVA demonstrated a significant effect of chronic treatment with 1BnTIQ $(F[2,30]=5.92$, $P<0.01)$, as well as acute L-DOPA administration $(F[2,30]=55.78, P<0.01)$ on the HVA concentration in the substantia nigra (Table 2). An interaction between 1BnTIQ and L-DOPA was also detected $(F[2,30]=5.92$, $P<0.01)$. The post hoc analysis showed that L-DOPA produced a massive increase in the HVA concentration (approximately $8,000 \% ; P<0.01$ ); this effect was partially blocked by chronic treatment with both concentrations of 1BnTIQ (Table 2).

L-DOPA $\quad$ significantly $\quad(F[2,30]=14.8, \quad P<0.01)$ affected the rate of DA metabolism measured as [HVA]/ [DA] (Table 2). The statistical analysis revealed no effect of chronic administration of $1 \mathrm{BnTIQ}$ on the rate of DA metabolism $(F[2,30]=0.1$, N.S. $)$ or interaction between 1 BnTIQ and L-DOPA $(F[2,30]=0.1$, N.S. $)$. Duncan's post hoc analysis demonstrated that acute treatment with L-DOPA strongly increased the rate of DA metabolism (approximately $800 \% ; P<0.01$ ); a similar effect was observed in the mixed groups when 1BnTIQ (25 and $50 \mathrm{mg} / \mathrm{kg}$ ) was coupled with acute L-DOPA (Table 2). 
Table 2 The effects of chronic administration of 1BnTIQ on L-DOPA-induced changes in DA metabolism in rat brain structures

\begin{tabular}{|c|c|c|c|c|c|c|}
\hline Treatment chronic & Treatment acute & DA (ng/g tissue) & DOPAC (ng/g tissue) & 3-MT (ng/g tissue) & HVA (ng/g tissue) & HVA/DA \\
\hline \multicolumn{7}{|l|}{ Substantia nigra } \\
\hline Saline & Saline & $564 \pm 44$ & $206 \pm 39$ & $37 \pm 2$ & $84 \pm 9$ & $15 \pm 2$ \\
\hline Saline & L-DOPA 100 & $5556 \pm 1015^{* *}$ & $25178 \pm 5309 * *$ & $181 \pm 24 * *$ & $6410 \pm 641 * *$ & $129 \pm 16^{* *}$ \\
\hline 1BnTIQ 25 & Saline & $502 \pm 23^{++}$ & $174 \pm 11^{++}$ & $40 \pm 3^{++}$ & $83 \pm 7^{++}$ & $17 \pm 2$ \\
\hline 1BnTIQ 50 & Saline & $413 \pm 16^{++}$ & $180 \pm 16^{++}$ & $32 \pm 2^{++}$ & $99 \pm 8^{++}$ & $24 \pm 2$ \\
\hline 1BnTIQ 25 & L-DOPA 100 & $1272 \pm 180^{+}$ & $2210 \pm 628^{++}$ & $89 \pm 11^{++}$ & $1864 \pm 514^{++}$ & $128 \pm 30^{* *}$ \\
\hline 1BnTIQ 50 & L-DOPA 100 & $3987 \pm 2414^{*}$ & $8142 \pm 4355^{++}$ & $142 \pm 45^{* *}$ & $4080 \pm 1394 * *^{+}$ & $181 \pm 30^{* *}$ \\
\hline Effect of 1BnTIQ & & $\begin{array}{l}F_{(2 / 30)}=2.08 \\
\text { N.S. }\end{array}$ & $\begin{array}{l}F_{(2 / 30)}=8.99 \\
P<0.01\end{array}$ & $\begin{array}{l}F_{(2 / 30)}=2.22 \\
\text { N.S. }\end{array}$ & $\begin{array}{l}F_{(2 / 30)}=5.92 \\
P<0.01\end{array}$ & $\begin{array}{l}F_{(2 / 30)}=0.1 \\
\text { N.S. }\end{array}$ \\
\hline Effect of L-DOPA & & $\begin{array}{l}F_{(2 / 30)}=12.64 \\
P<0.01\end{array}$ & $\begin{array}{l}F_{(2 / 30)}=25.72 \\
P<0.01\end{array}$ & $\begin{array}{l}F_{(2 / 30)}=34.24 \\
P<0.01\end{array}$ & $\begin{array}{l}F_{(2 / 30)}=55.78 \\
P<0.01\end{array}$ & $\begin{array}{l}F_{(2 / 30)}=14.8 \\
P<0.01\end{array}$ \\
\hline Interaction of $1 \mathrm{BnTI}$ & $\mathrm{IQ}+\mathrm{L}-\mathrm{DOPA}$ & $\begin{array}{l}F_{(2 / 30)}=2.00 \\
\text { N.S. }\end{array}$ & $\begin{array}{l}F_{(2 / 30)}=8.94 \\
P<0.01\end{array}$ & $\begin{array}{l}F_{(2 / 30)}=2.55 \\
\text { N.S. }\end{array}$ & $\begin{array}{l}F_{(2 / 30)}=5.92 \\
P<0.01\end{array}$ & $\begin{array}{l}F_{(2 / 30)}=0.1 \\
\text { N.S. }\end{array}$ \\
\hline \multicolumn{7}{|l|}{ Striatum } \\
\hline Saline & Saline & $9793 \pm 426$ & $1486 \pm 122$ & $383 \pm 16$ & $701 \pm 34$ & $7.2 \pm 0.3$ \\
\hline Saline & L-DOPA 100 & $25128 \pm 2755^{* *}$ & $33727 \pm 6080 * *$ & $269 \pm 16^{* *}$ & $9696 \pm 870 * *$ & $39.5 \pm 2.4 * *$ \\
\hline 1BnTIQ 25 & Saline & $7645 \pm 525^{++}$ & $1142 \pm 84^{++}$ & $410 \pm 21^{++}$ & $735 \pm 57^{++}$ & $9.9 \pm 1.1^{++}$ \\
\hline 1BnTIQ 50 & Saline & $6042 \pm 348^{+}$ & $1415 \pm 147^{++}$ & $335 \pm 14^{++}$ & $1111 \pm 104^{++}$ & $18.6 \pm 1.8^{* *++}$ \\
\hline 1BnTIQ 25 & L-DOPA 100 & $14653 \pm 3192^{++}$ & $4283 \pm 922^{++}$ & $330 \pm 15^{*+}$ & $3634 \pm 769^{*++}$ & $25.1 \pm 4.6^{* *++}$ \\
\hline 1BnTIQ 50 & L-DOPA 100 & $18281 \pm 5335^{* *}$ & $3318 \pm 550^{++}$ & $338 \pm 13^{++}$ & $7452 \pm 1903^{* *}$ & $42.6 \pm 2.3 * *$ \\
\hline Effect of 1BnTIQ & & $\begin{array}{l}F_{(2 / 30)}=2.94 \\
\text { N.S. }\end{array}$ & $\begin{array}{l}F_{(2 / 30)}=23.8 \\
P<0.01\end{array}$ & $\begin{array}{l}F_{(2 / 30)}=4.18 \\
P<0.05\end{array}$ & $\begin{array}{l}F_{(2 / 30)}=5.75 \\
P<0.01\end{array}$ & $\begin{array}{l}F_{(2 / 30)}=14.0 \\
P<0.01\end{array}$ \\
\hline Effect of L-DOPA & & $\begin{array}{l}F_{(2 / 30)}=25.54 \\
P<0.01\end{array}$ & $\begin{array}{l}F_{(2 / 30)}=36.4 \\
P<0.01\end{array}$ & $\begin{array}{l}F_{(2 / 30)}=23.67 \\
P<0.01\end{array}$ & $\begin{array}{l}F_{(2 / 30)}=66.71 \\
P<0.01\end{array}$ & $\begin{array}{l}F_{(2 / 30)}=138 \\
P<0.01\end{array}$ \\
\hline Interaction of $1 \mathrm{BnTI}$ & $\mathrm{IQ}+\mathrm{L}-\mathrm{DOPA}$ & $\begin{array}{l}F_{(2 / 30)}=1.13 \\
\text { N.S. }\end{array}$ & $\begin{array}{l}F_{(2 / 30)}=23.2 \\
P<0.01\end{array}$ & $\begin{array}{l}F_{(2 / 30)}=7.16 \\
P<0.01\end{array}$ & $\begin{array}{l}F_{(2 / 30)}=5.62 \\
P<0.01\end{array}$ & $\begin{array}{l}\mathrm{F}_{(2 / 30)}=5.9 \\
P<0.01\end{array}$ \\
\hline
\end{tabular}

1BnTIQ was chronic administered at two concentrations (25 and $50 \mathrm{mg} / \mathrm{kg}$ i.p.) during 14 consecutive days. In the mixed group, L-DOPA $(100 \mathrm{mg} / \mathrm{kg}$ i.p.) was given once, $15 \mathrm{~min}$ after last $1 \mathrm{BnTIQ}$ administration. Rats were decapitated $2 \mathrm{~h}$ after the injections. The results are expressed as the mean \pm SEM of six samples ( $n=6$ animals per group). Data were analyzed with a two-way ANOVA followed by Duncan's post hoc test. Statistical significance: $* P<0.05, * * P<0.01$ versus saline group; ${ }^{+} P<0.05,{ }^{++} P<0.01$ versus L-DOPA group

\section{Striatum}

In the striatum, a two-way ANOVA revealed no effect of chronic treatment with $1 \mathrm{BnTIQ}(F[2,30]=2.94$, N.S. $)$ on the DA concentration (Table 2). In contrast, the acute L-DOPA treatment produced a significant effect on the level of DA $(F[2,30]=25.54, \quad P<0.01)$. The statistical analysis revealed no interaction between chronic administration of 1 BnTIQ and L-DOPA administration $(F[2,30]=1.13$, N.S. $)$. The post hoc test indicated that acute doses of L-DOPA increased the level of DA (approximately $300 \%, P<0.01$ ) and that this effect was antagonized only by chronic treatment with a lower ( $25 \mathrm{mg} / \mathrm{kg}$ ) concentration of 1BnTIQ (Table 2).

A two-way ANOVA demonstrated a significant effect of chronic treatment with 1BnTIQ $(F[2,30]=23.8$, $P<0.01)$ as well as the acute administration of L-DOPA $(F[2,30]=36.4, P<0.01)$ on the DOPAC concentration in rat striatum (Table 2). An interaction between 1BnTIQ and L-DOPA was also detected $(F[2,30]=23.2$, $P<0.01)$. The post hoc analysis showed that L-DOPA produced a massive increase in DOPAC concentration (approximately 2,000\%; $P<0.01$ ); this effect was completely blocked by chronic treatment with both concentrations of 1BnTIQ (Table 2).

Chronic administration of 1BnTIQ $(F[2,30]=4.18$, $P<0.05)$ and acute administration of L-DOPA $(F[2,30]=23.67, P<0.01)$ both significantly affected the 3-MT concentration in striatum (Table 2). The statistical analysis also revealed an interaction between 1BnTIQ and L-DOPA $(F[2,30]=7.16, P<0.01)$. Duncan's post hoc test showed that L-DOPA decreased the level of 3-MT (approximately $30 \% ; P<0.01)$ and this effect was reversed by chronic treatment with both concentrations of 1BnTIQ (Table 2).

A two-way ANOVA revealed a significant effect of both the chronic 1BnTIQ treatment $(F[2,30]=5.75, P<0.01)$ and acute L-DOPA administration $(F[2,30]=66.71$, 
Table 3 The impact of acute and chronic administration of 1BnTIQ on L-DOPA-induced increase the concentration of 3-MDOPA in rat striatum

\begin{tabular}{|c|c|c|}
\hline Treatment acute & Treatment acute & 3-MDOPA (ng/g tissue) \\
\hline Saline & Saline & $1.5 \pm 0.19$ \\
\hline Saline & L-DOPA 100 & $48687 \pm 8801 * *$ \\
\hline 1BnTIQ 50 & Saline & $1.43 \pm 0.17$ \\
\hline 1BnTIQ 50 & L-DOPA 100 & $15125 \pm 3468^{++}$ \\
\hline \multicolumn{2}{|l|}{ Effect of 1BnTIQ } & $\begin{array}{l}F_{(1 / 18)}=7.38 \\
P<0.05\end{array}$ \\
\hline \multicolumn{2}{|l|}{ Effect of L-DOPA } & $\begin{array}{l}F_{(1 / 18)}=26.7 \\
P<0.01\end{array}$ \\
\hline \multicolumn{2}{|c|}{ Interaction of $1 \mathrm{BnTIQ}+\mathrm{L}-\mathrm{DOPA}$} & $\begin{array}{l}F_{(1 / 18)}=7.38 \\
P<0.05\end{array}$ \\
\hline Treatmentchronic & Treatmentacute & 3-MDOPA(ng/g tissue) \\
\hline Saline & Saline & $1.49 \pm 0.22$ \\
\hline Saline & L-DOPA 100 & $26868 \pm 3867 * *$ \\
\hline 1BnTIQ 25 & Saline & $1.52 \pm 0.24$ \\
\hline 1BnTIQ 50 & Saline & $1.17 \pm 0.12$ \\
\hline 1BnTIQ 25 & L-DOPA 100 & $8381 \pm 1714^{++}$ \\
\hline 1BnTIQ 50 & L-DOPA 100 & $18635 \pm 4868 * *+$ \\
\hline \multicolumn{2}{|l|}{ Effect of 1BnTIQ } & $\begin{array}{l}F_{(2 / 30)}=6.18 \\
P<0.01\end{array}$ \\
\hline \multicolumn{2}{|l|}{ Effect of L-DOPA } & $\begin{array}{l}F_{(2 / 30)}=69.78 \\
P<0.01\end{array}$ \\
\hline \multicolumn{2}{|c|}{ Interaction of 1BnTIQ + L-DOPA } & $\begin{array}{l}F_{(2 / 30)}=6.19 \\
P<0.01\end{array}$ \\
\hline
\end{tabular}

1BnTIQ was administered acute or chronic during 14 consecutive days at two concentrations ( 25 and $50 \mathrm{mg} / \mathrm{kg}$ i.p.). In the mixed group, L-DOPA (100 mg/kg i.p.) was given once, $15 \mathrm{~min}$ after 1BnTIQ administration. Rats were decapitated $2 \mathrm{~h}$ after the injections. The results are expressed as the mean \pm SEM $(n=5-6$ animals per group). Data were analyzed with a two-way ANOVA followed by Duncan's post hoc test. Statistical significance: * $P<0.05$, ** $P<0.01$ versus saline group; ${ }^{+} P<0.05,{ }^{++} P<0.01$ versus L-DOPA group

$P<0.01)$ on the HVA concentration in the striatum (Table 2). An interaction between the chronic administration of 1BnTIQ and L-DOPA was also detected $(F[2,30]=5.62, P<0.01)$. The post hoc analysis demonstrated that L-DOPA strongly increased the HVA concentration (approximately 1,400\%;P<0.01); this effect was partially antagonized only by chronic treatment with a lower $(25 \mathrm{mg} / \mathrm{kg})$ concentration of 1BnTIQ (Table 2).

Chronic administration with 1BnTIQ $(F[2,30]=14.0$, $P<0.01)$ and acute L-DOPA administration $(F[2,30]=$ $138, P<0.01)$ produced significant effects on the rate of DA metabolism measured as [HAV]/[DA] (Table 2). An interaction between $1 \mathrm{BnTIQ}$ and L-DOPA was also significant $(F[2,30]=5.9, \quad P<0.01)$. Duncan's post hoc analysis demonstrated that chronic treatment with a higher
$(50 \mathrm{mg} / \mathrm{kg})$ concentration of 1 BnTIQ and acute administration of L-DOPA induced an increase the rate of DA metabolism (approximately 300 and $600 \%$, respectively). Only a lower dose $(25 \mathrm{mg} / \mathrm{kg})$ of $1 \mathrm{BnTIQ}$ given chronically could partially antagonize this effect (Table 2).

\section{The Effect of Acute and Chronic Administration of $1 B n T I Q$ on L-DOPA Metabolism in Rat Striatum}

A two-way ANOVA demonstrated a significant effect of acute treatment with 1BnTIQ $(F[1,18]=7.38, P<0.05)$ or L-DOPA $(F[1,18]=26.7, P<0.01)$ on the 3 -MDOPA concentration in striatum (Table 3 ). An interaction between 1BnTIQ and L-DOPA was also detected $(F[1,18]=7.38$, $P<0.05)$. Duncan's post hoc test indicated that L-DOPA produced a massive increase the level of 3-MDOPA (by approx. 30,000 times; $P<0.01)$; this effect was strongly blocked (by approximately $70 \% ; P<0.01$ ) by acute treatment with 1 BnTIQ (50 mg/kg i.p.). Similarly, a twoway ANOVA revealed a significant effect of chronic administration of 1BnTIQ $(F[2,30]=6.18, P<0.01)$ and acute administration of L-DOPA $\quad(F[2,30]=69.8$, $P<0.01)$ on the concentration of 3-MDOPA in the striatum. Here again, the interaction between 1BnTIQ and L-DOPA was significant $(F[2,30]=6.19, P<0.01)$. The post hoc analysis demonstrated that L-DOPA strongly increased the 3-MDOPA concentration $(P<0.01)$; this effect was strongly inhibited by chronic treatment with a lower $(25 \mathrm{mg} / \mathrm{kg})$ concentration of 1BnTIQ (Table 3), while the higher concentration of 1 BnTIQ $(50 \mathrm{mg} / \mathrm{kg})$ antagonized this effect only partially $(P<0.05)$ (Table 3$)$.

\section{The Effect of Chronic Administration of $1 B n T I Q$ on the} L-DOPA-Induced Elevation of Caspase-3 Activity in Rat Hippocampus

Chronic systemic administration of 1BnTIQ at a concentration of $25 \mathrm{mg} / \mathrm{kg}$ did not alter caspase-3 activity in the hippocampus, whereas a higher concentration of 1BnTIQ $(50 \mathrm{mg} / \mathrm{kg})$ produced only a slight increase in this activity (Fig. 3). As expected, acute administration of L-DOPA $(100 \mathrm{mg} / \mathrm{kg}$ i.p. $)$ induced a significant increase (approx. $100 \%$; $P<0.01$ ) in the activity of caspase- 3 in the hippocampus. This effect was completely inhibited by multiple injections of $1 \mathrm{BnTIQ}$ at both concentrations $(P<0.01)$ (Fig. 3).

In Vivo Microdialysis

The Effects of a Single Administration of $1 B n T I Q$ on L-DOPA-Induced Changes in DA Release in Rat Striatum

A repeated one-way ANOVA revealed no effect of treatment on DA release into the extracellular space after acute 


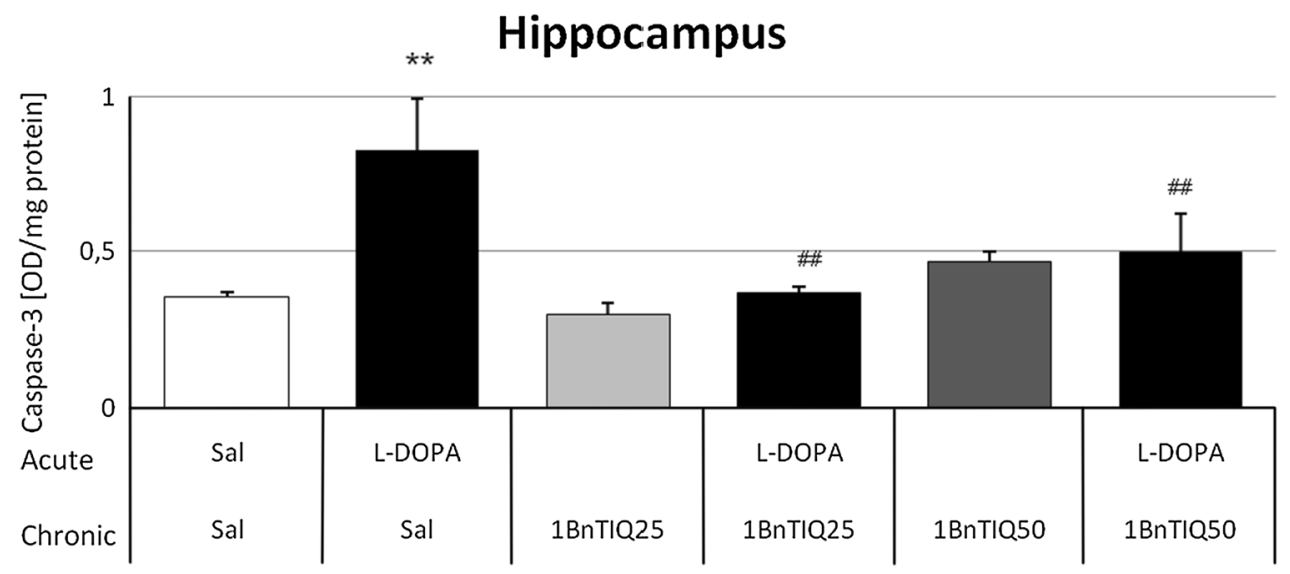

Fig. 3 The effect of 1BnTIQ on L-DOPA-induced increase in caspase-3 activity in rat hippocampus. 1BnTIQ was administered chronically at concentrations of 25 or $50 \mathrm{mg} / \mathrm{kg}$ i.p. for 14 consecutive days. In the mixed group, L-DOPA (100 mg/kg i.p.) was given once, $30 \mathrm{~min}$ after last 1BnTIQ administration. Rats were decapitated
$3 \mathrm{~h}$ after the last injection. The results are expressed as the mean $\pm \operatorname{SEM}$ ( $n=6$ animals per group). Data were analyzed with a two-way ANOVA, followed by Duncan's post hoc test. Statistical significance: $* * P<0.01$ versus saline-treated group; ${ }^{\# \#} P<0.01$ versus L-DOPA-treated group
Fig. 4 The effects of acute administration of 1BnTIQ on L-DOPA-induced changes in DA release (a) and 3-MT concentration (b) in rat striatum. Control samples were collected from " -60 " to " 0 ;" then, 1BnTIQ (50 mg/kg; at timepoint " 0 ") or L-DOPA (100 mg/kg; at timepoint " 40 ") was administered i.p. Dialysates were collected every $20 \mathrm{~min}$. In the mixed group, 1BnTIQ was injected $40 \mathrm{~min}$ before L-DOPA administration. The

concentration of DA (a) and its extraneuronal metabolite 3-MT

(b) was measured. The basal level of DA in striatum was $10.6 \pm 3.1 \mathrm{pg} / 20 \mu \mathrm{l}$. The data are expressed as the mean \pm SEM $(n=5-6)$.

Statistical significance:

$* P<0.05, * * P<0.01$ from the basal value (Duncan's test)
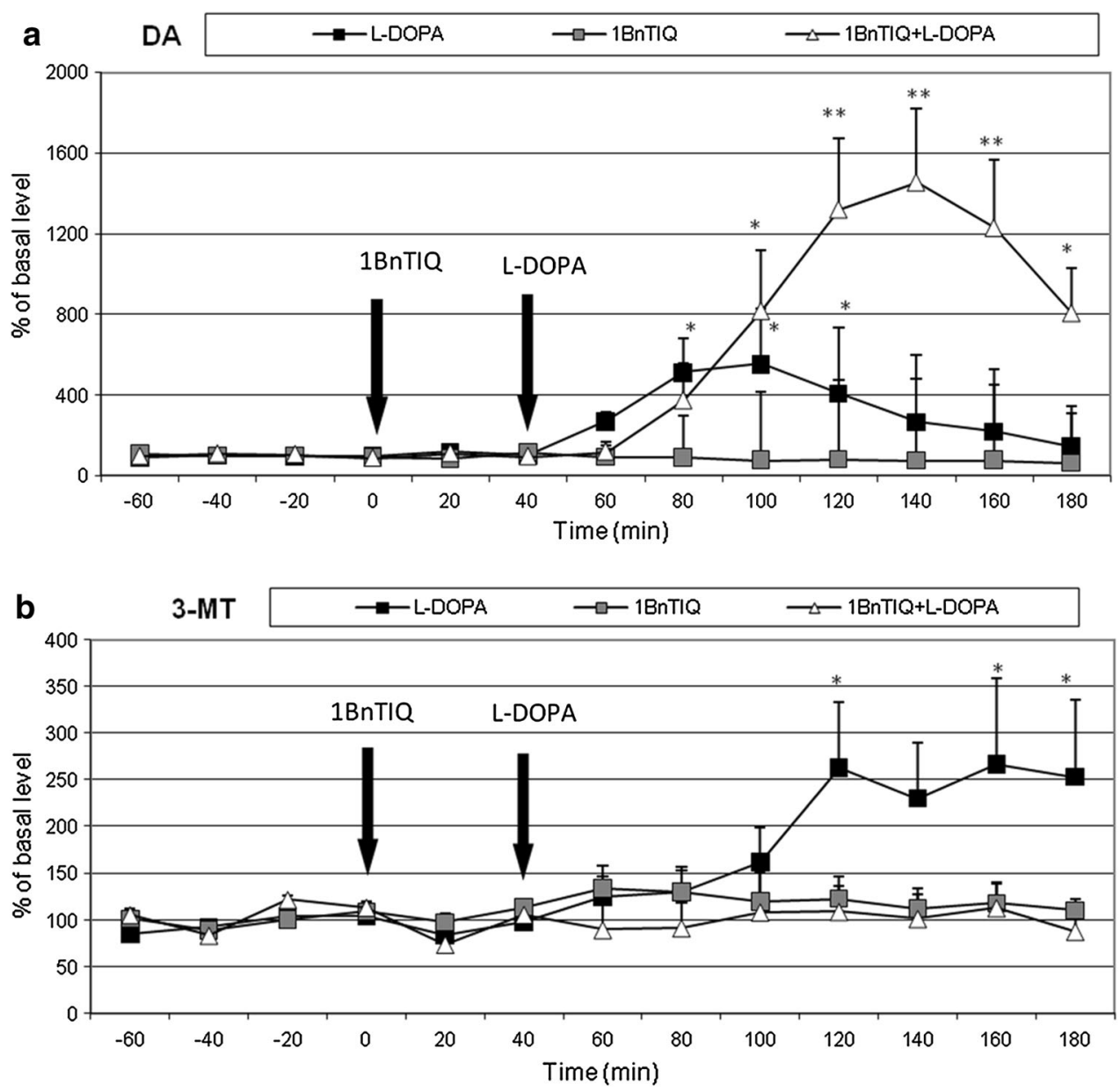

administration of either 1BnTIQ (50 mg/kg i.p.) or L-DOPA $(100 \mathrm{mg} / \mathrm{kg}$ i.p. $)(F[2,12]=3.03$, N.S. $)$. However, an effect of time $(F[12,144]=4.14, P<0.01)$ and an interaction between time and treatment were detected
$(F[24,144]=2.99, P<0.01)$. Duncan's post hoc analysis showed that acute L-DOPA administration produced a significant $(P<0.01)$ and long-lasting increase in DA release in the rat striatum (by approximately 500\%) 
Fig. 5 The effects of and chronic administration of 1BnTIQ on L-DOPA-induced changes in DA release (a) and 3-MT concentration (b) in rat striatum. 1BnTIQ was administered chronic at dose $50 \mathrm{mg} / \mathrm{kg}$ i.p. during 14 consecutive days. In the mixed group, L-DOPA $(100 \mathrm{mg} / \mathrm{kg}$ i.p.) was given once, $40 \mathrm{~min}$ after last 1BnTIQ administration. The control group was treated with saline. The dialysate was collected every $20 \mathrm{~min}$. The concentration of DA (a) and its extraneuronal metabolite 3-MT (b) was measured. The data are expressed as the mean \pm SEM $(n=5-6)$. Statistical significance: $* P<0.05$, $* * P<0.01$ from the basal value; ${ }^{+} P<0.05$ versus L-DOPA-treated group (Duncan's test)
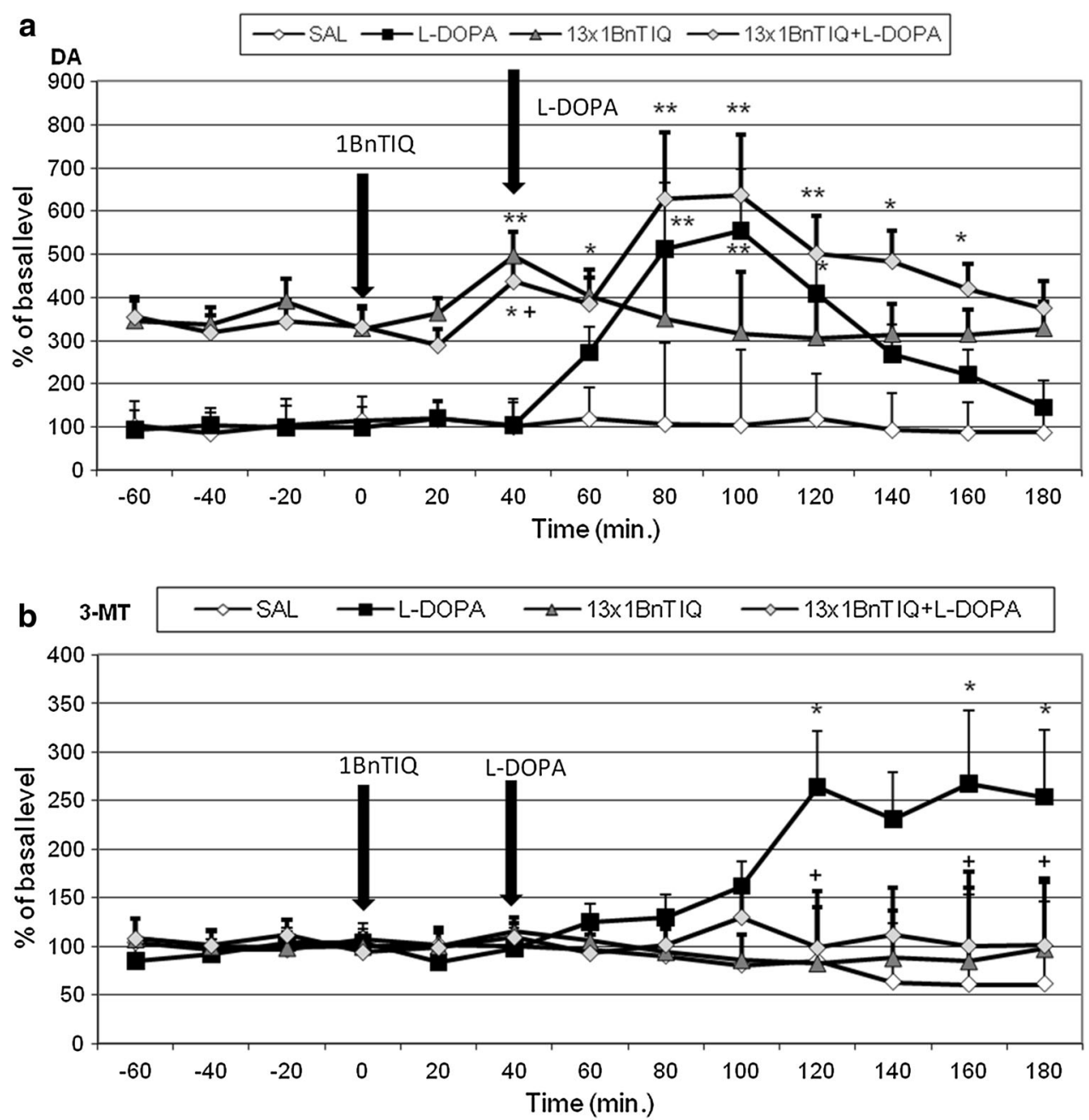

(Fig. 4a). 1BnTIQ alone did not produce a change in DA release. The post hoc test indicated that 1BnTIQ combined with L-DOPA potentiated the effect of L-DOPA and produced a long-lasting increase in the release of DA (up to $1,300 \%)(P<0.01)$ in the striatum (Fig. 4a).

A repeated one-way ANOVA did not reveal any effect of treatment on the concentration of 3-MT after a single injection of $1 \mathrm{BnTIQ}(50 \mathrm{mg} / \mathrm{kg}$ i.p.) or L-DOPA (100 $\mathrm{mg} / \mathrm{kg}$ i.p. $)(F[2,12]=1.15$, N.S. $)$. As for DA, an effect of time $(F[12,144]=2.09, P<0.05)$ was detected, but the interaction between time and treatment was not significant $(F[24,144]=0.98$, N.S. $)$. Duncan's test indicated that acute L-DOPA administration produced a significant $(P<0.05)$ increase in the concentration of $3-\mathrm{MT}$ in the rat striatum (up to $250 \%$ ) (Fig. 4b).

\section{The Effects of Multiple Administrations of $1 B n T I Q$ on $L^{-}$ DOPA-Induced Changes in DA Release in Rat Striatum}

A repeated two-way ANOVA showed a significant effect of chronic administration of 1BnTIQ (50 mg/kg i.p.)
$(F[1,18]=15.56, P<0.01)$ on DA release (treatment 1$),$ but the effect of acute dose of L-DOPA (treatment 2) was not significant (100 mg/kg i.p.) $(F[1,18]=3.1$, N.S. $)$. No interaction between treatment 1 and treatment 2 was found $(F[1,18]=0.27$, N.S.) (Fig. 4a). The statistical analysis demonstrated a significant effect of time $(F[12,216]=4.36$, $P<0.01)$, as well as an interaction between time and treatment $2(F[12,216]=5.24, P<0.01)$. However, there was no interaction between time and treatment 1 $(F[12,216]=0.81$, N.S. $)$ or between time, treatment 1 , and treatment $2(F[12,216]=0.23$, N.S.). Duncan's post hoc test indicated that acute L-DOPA administration produced a significant $(P<0.01)$ and long-lasting increase in DA release in the striatum (up to $500 \%$ ) (Fig. 5a).

A repeated two-way ANOVA showed that chronic administration of 1BnTIQ increased the release of DA in the rat striatum by up to $300 \%$ and, after the last dose, release of DA was elevated by an additional $100 \%$ (Fig. 5a). In the mixed group, chronic administration of 1BnTIQ weakened the effects of L-DOPA when compared to the group treated with L-DOPA alone (Fig. 5a). 
A repeated two-way ANOVA found no significant effect of treatment 1 after chronic administration of $1 \mathrm{BnTIQ}$ $(50 \mathrm{mg} / \mathrm{kg}$ i.p. $)(F[1,18]=0.66$, N.S. $)$ or of treatment 2 after an acute dose of L-DOPA $(100 \mathrm{mg} / \mathrm{kg}$ i.p.) on the concentration of 3 -MT $(F[1,18]=2.21$, N.S. $)$. Furthermore, there was no interaction between treatment 1 and treatment $2(F[1,18]=1.41$, N.S.) (Fig. $5 b)$. The statistical analysis showed no significant effect of time $(F[12,216]=0.57, \quad$ N.S. $), \quad$ time versus treatment 1 $(F[12,216]=0.96$, N.S. $)$, or time versus treatment 1 versus treatment $2(F[12,216]=1.42$, N.S.). However, there was a significant interaction between time and treatment 2 $(F[12,216]=1.98, P<0.05)$. Duncan's post hoc analysis showed that acute L-DOPA administration produced a significant $(P<0.05)$ elevation in the concentration of 3-MT (up to $250 \%$ ); this effect was completely antagonized by chronic administration of 1BnTIQ (Fig. 5b).

\section{Discussion}

The main finding of this study was that both acute and chronic systemic administrations of the endogenous neurotoxin 1BnTIQ disturbed the behavioral and biochemical effects of L-DOPA in the rat. Biochemical ex vivo studies have shown that administration of L-DOPA $(100 \mathrm{mg} / \mathrm{kg}$ i.p.), as a precursor of DA, causes a significant increase in DA metabolism, its concentration, and the concentration of all DA metabolites in the extrapyramidal brain structures substantia nigra and striatum. 1BnTIQ, at both investigated concentrations (25 and $50 \mathrm{mg} / \mathrm{kg}$ ), completely antagonized the effect of L-DOPA in the rat brain. Comparing the results of our behavioral test and our ex vivo and in vivo biochemical experiments, we observed that 1BnTIQ does not always act in the same direction. In the behavioral and ex vivo experiments, 1BnTIQ completely antagonized the L-DOPA-induced biochemical effects, while the in vivo microdialysis studies demonstrated the potentiation by 1BnTIQ of L-DOPA-evoked DA release in the striatum (specifically after acute administration of 1BnTIQ; Fig. 4a). Additionally, we have found that chronic administration of 1BnTIQ completely blocks the L-DOPAinduced increase in caspase-3 activity in the hippocampus.

DA was discovered as a neurotransmitter in the animal brain based on its specific regional distribution. 1BnTIQ, like reserpine, a specific inhibitor of vesicular monoamine uptake transporter (VMAT2), produces depletion of striatal DA, such that even synthesized DA is not stored in vesicles efficiently (Wasik et al. 2009). Reserpine is a model substance frequently used to produce parkinsonism in animals (Colpaert 1987; Lorenc-Koci et al. 1995). Dysfunction of DA neurons has been explicitly correlated with damage to basic transport and storage mechanisms of neurotransmitters [neuronal (DAT) and vesicular (VMAT2) uptake]. The above findings suggest that 1BnTIQ may damage the VMAT2 in dopaminergic neurons, leading to the pathological release of DA into the cytosol. L-DOPA therapy supplements DA deficiencies and increases its metabolism in the brain. Peripheral coadministration of aromatic L-amino acid decarboxylase (AADC) inhibitors (carbidopa, benserazid) improves the bioavailability of L-DOPA (Bartholini et al. 1967). Exogenously administered L-DOPA may be taken up into DA terminals in the brain by the DA reuptake transporter DAT and may be decarboxylated to DA, primarily within AADC-containing cells in the striatum. Therefore, the undisturbed function of DAT is a crucial factor for increasing DA metabolism and the therapeutic effectiveness of L-DOPA in the clinic. Our previous studies, carried out in rat striatum slices, demonstrated that 1BnTIQ significantly inhibits (IC50 in low micromolar concentrations) the DA reuptake transporter (Patsenka et al. 2004). A similar effect was shown by Okada et al. (1998), where 1BnTIQ inhibited the uptake of $\left[{ }^{3} \mathrm{H}\right]$ dopamine through the DAT expressed in HEK293 cells. In light of these findings, DAT is a likely candidate responsible for the selective transport of 1BnTIQ into dopaminergic neurons, leading to the 1BnTIQ neurotoxicity correlated with impairment of DA storage by inhibition of VMAT2 (Wasik et al. 2009).

Our biochemical ex vivo studies showed that administration of L-DOPA (100 mg/kg i.p.) causes an increase in DA and all its metabolites (DOPAC, 3-MT, and HVA) both in the substantia nigra and in the striatum (Table 1). 1BnTIQ at both concentrations (25 and $50 \mathrm{mg} / \mathrm{kg}$ i.p.) decreased DA in the nigrostriatal structures (Table 1). In the mixed groups, L-DOPA-induced effects were completely inhibited by both single and multiple administrations of 1BnTIQ. The differences between acute and chronic administration that we observed suggest that, during chronic administration of 1BnTIQ, some tolerance to its DA-depressing effect develops, while the impairment of DA synthesis continues. On the other hand, weakening in the inhibitory action 1BnTIQ after repeated administration may indicate adaptation in the brain and/or brain plasticity. Our ex vivo studies suggest that 1BnTIQ prevents the uptake of L-DOPA into the neuron and/or blocks the conversion of L-DOPA into DA. As mentioned, 1BnTIQ antagonism of L-DOPA-evoked increase in DA metabolism could be linked to DAT inhibition by 1BnTIQ (Okada et al. 1998; Patsenka et al. 2004). In fact, the in vivo microdialysis results discussed in this paper support this hypothesis. Our results show that 1BnTIQ alone (50 $\mathrm{mg} / \mathrm{kg}$ i.p.) does not produce significant changes in DA release (Fig. 4a). However Katagiri et al. (2009) reported that acute $1 \mathrm{BnTIQ}$ (40 $\mathrm{mg} / \mathrm{kg}$ i.p.) injection produced a weak increase in the DA release. The discrepancies 


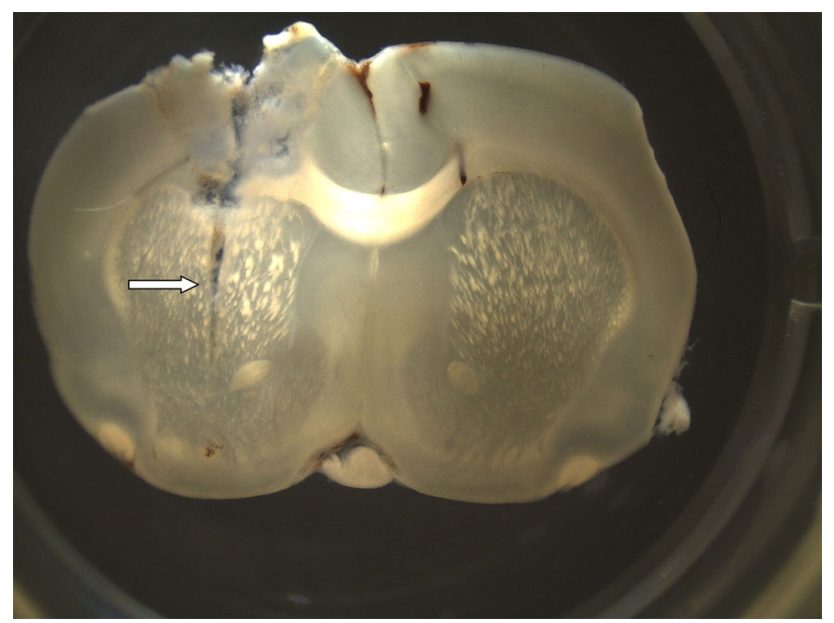

Fig. 6 The implantation of the cannula in the rat striatum

between our results may be explained through the different areas of the cannula implantation. While our dialysates were collected from central part of the striatum (Fig. 6); in the case of Katagiri et al. (2009) it was done from part of the ventral striatum.

L-DOPA is a direct substrate for COMT, and its metabolism leads to the production of 3-O-methyl-dopa (3-MDOPA) in the brain. Therefore, COMT is responsible both for the metabolic inactivation of extraneuronal DA to 3-MT and for the metabolism of L-DOPA to 3-MDOPA (Kopin 1985; Antkiewicz-Michaluk et al. 2001). Selective COMT inhibitors, such as entacapone, tolcapone, and CGP28014, effectively block the O-methylation of L-DOPA and the formation of 3-MDOPA, thus improving its bioavailability and brain penetration (Kaakkola et al. 1994). COMT inhibitors are used as an adjunctive treatment for PD as they increase central L-DOPA availability (Mannisto and Kaakkola 1999). Our ex vivo and in vivo studies clearly support the notion that $1 \mathrm{BnTIQ}$ inhibits COMT-activity, as it significantly decreased the concentrations of both the L-DOPA and DA metabolites, 3-MDOPA and 3-MT, respectively (Table 3; Figs. 4b, 5b).

1BnTIQ given acutely $40 \mathrm{~min}$ before L-DOPA (100 $\mathrm{mg} / \mathrm{kg}$ i.p.) administration, augmented L-DOPA effect and was produced an additional increase in the extraneuronal DA concentration (up to $1,300 \% ; P<0.01$ ) in striatum (Fig. 4a). However, chronic administration of 1BnTIQ clearly weakens this increase in the L-DOPA effect, likely as a result of the tolerance development (Fig. 5a). The effect of 1BnTIQ connected with the COMT inhibition presented in the paper and discussed above can explained the potentiation of L-DOPA-produced an increase of the extraneuronal DA concentration in microdialysis study (Figs. 4a, 5a).

It is well known that although L-DOPA is the gold standard for the treatment of PD symptoms. L-DOPA can lead to adverse effects, either as a result of oxidative stress because it has the potential to auto-oxidize to a quinine derivative, generating ROS and depleting striatal GSH (Ogawa et al. 1994; Spencer et al. 1996; Hattoria et al. 2009), or by inducing apoptosis, as evidenced by the increased activity of caspase-3 and DNA damage via a mechanism independent of oxidative stress (Pedrosa and Soares-da-Silva 2002; Tanaka and Ogawa 2005; Emdadul Haque et al. 2003). We confirmed these findings with one of our ex vivo experiments: acute L-DOPA injection produced a significant increase in caspase- 3 activity, an effect that was completely inhibited by chronic administration of 1BnTIQ at both tested concentrations (Fig. 3). It appears that we are observing artificial "neuroprotective" effects of 1BnTIQ, related to its inhibition of DAT activity, and thus of L-DOPA neuronal uptake.

We know from our previous in vitro study that 1BnTIQ, at micromolar concentrations, has neurotoxic activity in rat hippocampal cultures, in which it increases apoptotic markers (caspase-3 activity and LDH release; Wąsik et al. 2014). However, follow-up ex vivo studies suggested that chronic administration of 1BnTIQ did not have neurotoxic effects on dopaminergic neurons in the substantia nigra, as assessed by tyrosine hydroxylase activity and the level of alpha-synuclein (Wassik et al. 2014). In contrast, in vitro experiments carried out in human dopaminergic cells showed that 1BnTIQ increased alpha-synuclein expression and caused nuclear damage (Shavali et al. 2004).

The results obtained from our behavioral studies are in agreement with the results of the biochemical ex vivo analysis in demonstrating both acute and chronic 1BnTIQ antagonisms of L-DOPA-induced locomotor hyperactivity in rat (Figs. 1a, b, 2a, b). However, in the combined groups, stereotyped behaviors linked to L-DOPA administrations, such as sniffing and sometimes licking were still present.

In conclusion, our results demonstrate that $1 \mathrm{BnTIQ}$ is not only an endogenous neurotoxin that disrupts the activity of dopaminergic neurons but is also an antagonist of L-DOPA-evoked behavioral and biochemical effects. The data from our ex vivo biochemical analysis and in vivo microdialysis study suggest that the molecular mechanism of action of 1BnTIQ may involve its inhibition of either or both DAT and COMT activities in the brain. Furthermore, elevated endogenous levels of 1BnTIQ may cause serious adverse effects in PD patients undergoing of L-DOPA therapy.

Acknowledgments Thanks are due to Dr. Jan Boksa (Department of Medicinal Chemistry, Institute of Pharmacology PAS, Krakow, Poland) for the synthesis of 1BnTIQ. We are also gratefully acknowledge the technical assistance of Maria Kafel and Krzysztof Michalski. This study was supported by the Polish Committee of Scientific Research, Grant No. N N401 004836, and by statutory 
funds of the Institute of Pharmacology, Polish Academy of Sciences, Krakow, Poland.

Open Access This article is distributed under the terms of the Creative Commons Attribution License which permits any use, distribution, and reproduction in any medium, provided the original author(s) and the source are credited.

\section{References}

Antkiewicz-Michaluk L, Michaluk J, Mokrosz M, Romańska I, Lorenc-Koci E, Otha S, Vetulani J (2001) Different action on dopamine catabolic pathways of two endogenous 1,2,3, 4-tetrahydroisoquinolines with similar antidopaminergic properties. J Neurochem 78:100-108

Bartholini G, Burkard WP, Pletscher A, Bates HM (1967) Increase of cerebral catecholamines caused by 3,4-dihydroxyphenylalanine after inhibition of peripheral decarboxylase. Nature 215:852-853

Bayir H, Kapralovv AA, Jiang J, Huang Z, Tyurina YY, Tyurin VA, Zhao Q, Belikova NA, Vlasova II, Maeda A, Zhu J, Na HM, Mastroberardino PG, Sparvero LJ, Amoscato AA, Chu CT, Greenamyre JT, Kagan VE (2009) Peroxidase mechanism of lipid-dependent cross-linking of synuclein with cytochrome $c$ : protection against apoptosis versus delayed oxidative stress in Parkinson disease. J Biol Chem 284(23):15951-15969

Cannon JG, Webster GL (1957) Syntheses of isoquinoline derivatives using 1-(lithi methyl) isoquinoline. J Am Pharm Assoc Am Pharm Assoc 47(5):353-355

Colpaert FC (1987) Pharmacological characteristics of tremor, rigidity and hypokinesia induced by reserpine in rat. Neuropharmacology 26:1431-1440

Emdadul Haque M, Asanuma M, Higashi Y, Miyazaki I, Tanaka K, Ogawa N (2003) Apoptosis-inducing neurotoxicity of dopamine and its metabolites via reactive quinine generation in neuroblastoma cells. Biochim Biophys Acta 1619:39-52

Filip M, Antkiewicz-Michaluk L, Zaniewska M, Frankowska M, Gołda A, Vetulani J, Przegaliński E (2007) Effects of 1-methyl1,2,3,4-tetrahydroisoquinoline on the behavioral effects of cocaine in rats. J Physiol Pharmacol 58:625-639

Gandhi S, Wood NW (2005) Molecular pathogenesis of Parkinson's disease. Hum Mol Genet 14:2749-2755

Hanrott K, Gudmunsen L, O'Neill MJ, Wonnacott S (2006) 6-Hydroxydopamine-induced apoptosis is mediated via extracellular auto-oxidation and caspase-3-dependent activation of protein kinase c-delta. J Biol Chem 281(9):5373-5382

Hattoria N, Wanga M, Taka H, Fujimura T, Yoritaka A, Kubo S, Mochizuki H (2009) Toxic effects of dopamine metabolism in Parkinson's disease. Parkinsonism Relat Disord 15(Suppl 1):35-38

Kaakkola S, Gordin A, Mannisto PT (1994) General properties and clinical possibilities of new selective inhibitors of catechol $O$-methyltransferase. Gen Pharmacol 25:813-824

Kajta M, Wójtowicz A, Maćkowiak M, Lasoń W (2009) Aryl hydrocarbon receptor-mediated apoptosis of neural cells: a possible interaction with estrogen receptor signaling. Neuroscience 158:811-822

Katagiri N, Abe K, Kitabatake M, Utsunomiya I, Horiguchi Y, Hoshi K, Taguchi K (2009) Single administration of 1-benzyl-1,2,3, 4-tetrahydroisoquinoline increases the extracellular concentration of dopamine in rat striatum. Neuroscience 160:820-828

Klockgether T (2004) Parkinson's disease: clinical aspects. Cell Tissue Res 318:115-120
Kohta R, Kotake Y, Hosoya T, Hiramatsu T, Otsubo Y, Koyama H, Hirokane Y, Yokoyama Y, Ikeshoji H, Oofusa K, Suzuki M, Ohta S (2010) 1Benzyl-1,2,3,4-tetrahydroisoquinoline binds with tubulin beta, a substrate of parkin, and reduces its polyubiquitination. J Neurochem 114:1291-1301

Kopin IJ (1985) Catecholamine metabolism: basic aspects and clinical significance. Pharmacol Rev 37:333-364

Kotake Y, Tasaki Y, Makino Y, Otha S, Hirobe M (1995) 1-Benzyl1,2,3,4-tetrahydroisoquinoline as a parkinsonism-inducing agent: a novel endogenous amine in mouse brain and parkinsonian CSF. J Neurochem 65(6):2633-2638

Kotake Y, Ohta S, Kanazawa I, Sakurai M (2003) Neurotoxicity of an endogenous brain amine, 1-benzyl-1,2,3,4-tetrahydroisoquinoline, in organotypic slice co-culture of mesencephalon and striatum. Neuroscience 117:63-70

Lorenc-Koci E, Ossowska K, Wardas J, Wolfarth S (1995) Does reserpine induce parkinsonian rigidity? J Neural Transm Park Dis Dement Sect 9:211-223

Mannisto PT, Kaakkola S (1999) Catechol- $O$-methyltransferase (COMT): biochemistry, molecular biology, pharmacology, and clinical efficacy of the new selective COMT inhibitors. Pharmacol Rev 51:593-628

Moore DJ, West AB, Dawson VL, Dawson TM (2005) Molecular pathophysiology of Parkinson's disease. Annu Rev Neurosci 28:57-87

Nicholson DW, Ali A, Thornberry NA, Vaillancourt JP, Ding CK, Gallant M, Gareau Y, Griffin PR et al (1995) Identification and inhibition of the ICE/CED-3 protease necessary for mammalian apoptosis. Nature 376:37-43

Ogawa N, Asanuma M, Kondo Y, Kawada Y, Yamamoto M, Mori A (1994) Differential effects of chronic L-DOPA treatment on lipid peroxidation in the mouse brain with or without pretreatment with 6-hydroxydopamine. Neurosci Lett 17:55-58

Okada T, Shimada S, Sato K, Kotake Y, Kawai H, Ohta S, Tohyama M, Nishimura T (1998) Tetrahydropapaveroline and its derivatives inhibit dopamine uptake through dopamine transporter expressed in HEK293 cells. Neurosci Res 30:87-90

Patsenka A, Michaluk J, Antkiewicz-Michaluk L (2004) 1,2,3, 4-Tetrahydroisoquinoline alkaloids as endogenous inhibitors of brain monoamine oxidase, tyrosine hydroxylase and uptake of monoamines: in vitro study. In: 13th international symposium on molecular and physiological aspects of regulatory processes of the organism, Kraków, Poland, Materials 2004, p 344

Pedrosa R, Soares-da-Silva P (2002) Oxidative and non-oxidative mechanisms of neuron al cell death and apoptosis by L-3, 4-dihydroxyphenylalanine (L-DOPA) and dopamine. Br J Pharmacol 137:1305-1313

Sas K, Robotka H, Toldi J, Vecsei L (2007) Mitochondria, metabolic disturbances, oxidative stress and the kynurenine system, with focus on neurodegenerative disorders. J Neurol Sci 257:221-239

Shavali S, Ebadi M (2003) 1-Benzyl-1,2,3,4-tetrahydroisoquinoline (1BnTIQ), an endogenous neurotoxin, induces dopaminergic cell death through apoptosis. Neurotoxicology 24:417-424

Shavali S, Carlson EC, Swinscoe JC, Ebadi M (2004) 1-Benzyl1,2,3,4-tetrahydroisoquinoline, a Parkinsonism-inducing endogenous toxin, increases \& \#x03B1;-synuclein expression and causes nuclear damage in human dopaminergic cells. J Neurosci Res 76:563-572

Spencer JPE, Jenner A, Butler J, Aruoma OI, Dexter DT, Jenner P, Halliwell B (1996) Evaluation of the pro-oxidant and antioxidant actions of L-DOPA and dopamine in vitro: implications for Parkinson's disease. Free Radic Res 24:95-105

Tanaka K, Ogawa N (2005) Dopamine agonist cabergoline inhibits levodopa-induced caspase activation in 6-OHNA-lesioned mice. Neurosci Res 51:9-13 
Wąsik A, Romańska I, Antkiewicz-Michaluk L (2009) 1-Benzyl1,2,3,4-tetrahydroisoquinoline, an endogenous parkinsonisminducing toxin, strongly potentiates MAO-dependent dopamine oxidation and impairs dopamine release: ex vivo and in vivo neurochemical studies. Neurotox Res 15:23-31
Wąsik A, Kajta M, Lenda T, Antkiewicz-Michaluk L (2014) Concentration-dependent opposite effects of 1-benzyl-1,2,3, 4-tetrahydroisoquinoline on markers of apoptosis: in vitro and ex vivo studies. Neurotox Res 25:90-99 